\title{
Time and frequency domain estimates of spontaneous baroreflex sensitivity provide early detection of autonomic dysfunction in diabetes mellitus
}

\author{
A. Frattola ${ }^{2}$, G. Parati ${ }^{2,3}$, P. Gamba ${ }^{1}$, F. Paleari ${ }^{1}$, G. Mauri ${ }^{1}$, M. Di Rienzo ${ }^{4}$, P. Castiglioni ${ }^{4}$, G. Mancia ${ }^{1,3}$ \\ ${ }^{1}$ Cattedra di Medicina Interna Università di Milano and Ospedale S.Gerardo Monza, Italy \\ ${ }^{2}$ Istituto Scientifico Ospedale S. Luca, Istituto Auxologico Italiano, Milano, Italy \\ ${ }^{3}$ Centro Fisiologia Clinica e Ipertensione, IRCCS, Ospedale Maggiore, Milano, Italy \\ ${ }^{4}$ LaRC, Centro di Bioingegneria, Fondazione Pro Juventute, Milano, Italy
}

\begin{abstract}
Summary Diabetic autonomic dysfunction is associated with a high risk of mortality which makes its early identification clinically important. The aim of our study was to compare the detection of autonomic dysfunction provided by classical laboratory autonomic function tests with that obtained through computer assessment of the spontaneous sensitivity of the baroreceptor-heart rate reflex (BRS) by time domain and frequency domain techniques. In 20 normotensive diabetic patients (mean age \pm SD $41.9 \pm 8.1$ years) with no evidence of autonomic dysfunction on laboratory autonomic testing (D0) blood pressure (BP) and ECG were continuously monitored over $15 \mathrm{~min}$ in the supine position. BRS was assessed as the slope of the regression line between spontaneous increases or reductions in systolic BP and linearly related lengthening or shortening in RR interval over sequences of at least 4 consecutive beats (sequence method), or as the squared ratio between RR interval and systolic BP spectral powers around $0.1 \mathrm{~Hz}$. We compared the results with those of 32 age-matched normotensive diabetic patients with abnormal autonomic function tests (D1) and with those of
\end{abstract}

24 healthy age-matched control subjects with normal autonomic function tests (C). Compared to C, BRS was markedly less in D1 when assessed by both the slope of the two types of sequences (data pooled) and by the spectral method $(-71.3 \%$ and $-60.2 \%$ respectively, both $p<0.01)$. However, BRS was consistently although somewhat less markedly reduced in D0, the reduction being clearly evident for all the estimates $(-57.0 \%$ and $-43.5 \%$, both $p<0.01)$. The effects were more evident than those obtained by the simple quantification of the RR interval variability. These data suggest that time and frequency domain estimates of spontaneous BRS allow earlier detection of diabetic autonomic dysfunction than classical laboratory autonomic tests. The estimates can be obtained by short non-invasive recording of the BP and RR interval signals in the supine patient, i.e. under conditions suitable for routine outpatient evaluation. [Diabetologia (1997) 40: 1470-1475]

Keywords Diabetes mellitus, autonomic nervous system, arterial baroreflex, blood pressure variability, heart rate variability, blood pressure.
Diabetic autonomic dysfunction, even when not yet manifest, is associated with a high risk of mortality [1-11] which makes its early identification clinically important. In the early eighties Ewing and co-workers $[1,12]$ validated a battery of laboratory tests for

Received: 30 April 1997 and in revised form: 31 July 1997

Corresponding author: Professor G. Mancia, Cattedra di Medicina Interna, Ospedale S. Gerardo, via Donizetti, I-20052 Monza, Italy

Abbreviations: SBP, Systolic blood pressure. identification of autonomic abnormalities in patients with diabetes mellitus. These tests consist in the measurement of the heart rate changes induced by manouvers such as deep breathing, Valsalva and standing which engage reflexes that alter vagal and sympathetic modulation of the heart [13-17]. They further consist in the measurement of the blood pressure responses to standing, cold pressure test and hand-grip exercise which allow assessment of sympathetic modulation of systemic vascular resistance [15-19].

Although useful for the identification of a diabetic-dependent damage of autonomic cardiovascular 
control, the "Ewing" tests are characterized by a suboptimal reproducibility [20]. Furthermore, their sensitivity is limited, particularly when blood pressure responses are measured. Finally, these tests require an often uneasy co-operation by the patients. To overcome these problems power spectral analysis of frequency components of heart rate variability that are believed to reflect autonomic cardiac modulation is increasingly used $[21,22]$. However, the spectral approach only allows a more sensitive detection of cardiac autonomic abnormalities if associated with manouvers that require a change in breathing pattern [23], or active or passive postural changes, which is often not easy to perform in the clinical setting.

In the last few years methods have been developed that allow neural modulation of the sinus node by arterial baroreceptors to be assessed by computer analysis of the relationship between spontaneous changes in blood pressure and pulse interval in the time and frequency domain [24-28]. The aim of our study was to determine whether: 1 ) these methods also allow early identification of autonomic cardiac abnormalities in diabetes mellitus; and 2) this can be obtained in conditions most favourable to the clinical setting, i.e. in patients resting supine for only $15 \mathrm{~min}$.

\section{Subjects and methods}

Subjects. Our study included 52 diabetic subjects of either sex between the ages of 22 and 54 years (mean \pm SD $42.9 \pm$ 9.7 years) who consecutively attended the diabetes clinic of the S. Gerardo Hospital and who had the following characteristics. 1) Body mass index never greater than normal values by more than $20 \%$. 2) No major disease beside diabetes. 3) No clinical manifestation of cardiac and vascular disease. 4) No abnormality in renal function; and 5) no treatment with antihypertensive or other cardiovascular drugs. In 28 patients diabetes was insulin-dependent and in the remaining 24 patients non-insulin-dependent. Diabetic complications were found in 26 patients which consisted of retinopathy $(n=7$, fluoroangiography), microalbuminuria ( 3 urine samples in the preceding year) and somatic neuropathy $(n=11$, nerve conduction studies). All subjects were under treatment with insulin and/or oral antidiabetic agents and their metabolic control was satisfactory, based on $\mathrm{HbA}_{1 \mathrm{c}}$ or serum glucose values. Blood pressure was normal (i.e. $<140 / 90 \mathrm{mmHg}$, systolic/diastolic) at sphygmomanometer measurements obtained in the outpatient clinic. Heart rate (palpatory method over $1 \mathrm{~min}$ ) was also normal ( $<90$ beats per min). We used 24 normotensive agematched healthy subjects, selected from among hospital staff, as controls. These subjects were 18 males and 6 females. Their mean age $\pm S D$ was $40.3 \pm 6.3$ years. Their blood pressure was $123.3 \pm 3.3 \mathrm{mmHg}$ systolic and $80.1 \pm 2.7 \mathrm{mmHg}$ diastolic, and their heart rate was $73.5 \pm 1.7$ beats per min.

Traditional evaluation of autonomic neuropathy. The presence of autonomic neuropathy was assessed by three "Ewing" tests: 1) RR interval response to deep breathing; 2) RR interval response to standing and 3) blood pressure response to standing. The RR interval response to deep breathing was measured by: a) asking the supine subject to breathe deeply at a rate of 6 breaths per min while monitoring the ECG via a standard lead; and b) taking the mean difference between the maximal RR interval value recorded during three successive breathing cycles. The RR interval response to standing was measured by: a) asking the subject to lie quietly supine for 4 min and quickly stand up during ECG monitoring; and b) taking the ratio between the change in RR interval occurring 15 and 30 s from assumption of the upright posture, i. e. the so-called 30:15 ratio that describes the shortening in RR interval that occurs early after shifting from lying to standing and the subsequent return towards baseline RR interval values. The blood pressure response to standing was measured by mercury sphygmomanometry after the subject had been standing for 1 min following a lying period of $4 \mathrm{~min}$. The response to each test was assessed as reported in literature [17]. Namely, in subjects aged 40 to 44 years the deep breathing test was regarded as abnormal or borderline if the ratio between the maximal and minimal RR interval was less than 1.17 or between 1.17 and 1.20 , respectively. The RR interval response to standing was regarded as abnormal or borderline if the 30:15 ratio was less than 1.90 or between 1.90 and 2.12, respectively. All RR interval criteria were reduced by a factor of two for each 5-year increment of age. The blood pressure response to standing was regarded as abnormal or borderline if at all ages the systolic blood pressure fall on standing was more than $29 \mathrm{mmHg}$ or between 29 and $10 \mathrm{mmHg}$, respectively. A normal response was graded as 0 , a borderline response as 1 and an abnormal response as 2 . In each subject the scores assigned to each individual test were summed to obtain a total score that ranged from 0 to 6 . According to the total score the diabetic patients were divided into two groups. Namely, 20 patients with a total score equal to 0 (age $41.9 \pm 8.1$ years) were regarded as having no evidence of autonomic neuropathy (D0). The remaining 32 patients (age $43.6 \pm 9.2$ years) with a total score equal or greater than 1 were regarded as having autonomic neuropathy. The demographic, haemodynamic and clinical characteristics of the control subjects, the D0 patients and the D1 patients are shown in Table 1, together with the reference to their conventional autonomic neuropathy score.

Computerized assessment of baroreceptor-heart rate reflex. Two hours after a light meal all control and diabetic subjects underwent a beat-to-beat blood pressure and ECG recording (1 standard lead) for $15 \mathrm{~min}$ in the supine position. Blood pressure was recorded non-invasively by a Finapres device (Finapres, Ohmeda, Englewood, Colo., USA), which is known to reliably track both fast and slow changes in intra-arterial blood pressure $[29,30]$. As described in detail in previous papers [29-31] the blood pressure signal was A/D converted, sampled at $200 \mathrm{~Hz}$, digitized at 12 bit resolution by an Olivetti XP5 computer and edited from morphological aberrances such as ectopic beats, movement artifacts and adjustments of the volume-clamp set point by an interactive procedure. From the edited signal systolic blood pressure was derived for each pulse wave. The same procedure was employed for the RR interval signal except that the sampling rate was $600 \mathrm{~Hz}$. The beat-tobeat systolic blood pressure and RR interval values were stored in separate time series. Average systolic blood pressure and RR interval values were computed for the whole $15 \mathrm{~min}$, together with the corresponding standard deviations which were taken as measures of their variability.

In addition, identification was obtained of the sequences of four or more consecutive beats during which systolic blood pressure (SBP) and RR interval showed a spontaneous progressive increase $(+\mathrm{RR} /+\mathrm{SBP})$ or a spontaneous progressive reduction $(-\mathrm{RR} / \mathrm{SBP})$, the step-wise changes being at least $1 \mathrm{mmHg}$ and $6 \mathrm{~ms}$, respectively. The regression coefficient (or slope) of the sequence was taken as a measure of the sensitivity 
Table 1. Demographic, haemodynamic and metabolic characteristics of control subjects $(\mathrm{C})$ and of the patients with no evidence $\left(D_{0}\right)$ or evidence $\left(D_{1}\right)$ of autonomic neuropathy

\begin{tabular}{|c|c|c|c|}
\hline Variable & $\mathrm{C}$ & D 0 & D 1 \\
\hline $\begin{array}{l}\text { Autonomic function } \\
\text { tests (total score) }\end{array}$ & 0 & 0 & $\geq 1$ \\
\hline$n$ (male/female) & $2418 / 6$ & $2013 / 7$ & $3213 / 19$ \\
\hline Age (years) & $40.3 \pm 6.3$ & $41.9 \pm 8.1$ & $43.6 \pm 9.2$ \\
\hline $\begin{array}{l}\text { Systolic blood } \\
\text { pressure }(\mathrm{mm} \mathrm{Hg})\end{array}$ & $123.3 \pm 3.3$ & $128.9 \pm 4.9$ & $131.1 \pm 5.3$ \\
\hline $\begin{array}{l}\text { Diastolic blood } \\
\text { pressure }(\mathrm{mm} \mathrm{Hg})\end{array}$ & $80.1 \pm 2.7$ & $79.0 \pm 2.8$ & $80.2 \pm 3.6$ \\
\hline $\begin{array}{l}\text { Heart rate } \\
\text { (beats/min) }\end{array}$ & $73.5 \pm 1.7$ & $75.4 \pm 2.1$ & $73.3 \pm 2.3$ \\
\hline $\begin{array}{l}\text { Serum creatinine } \\
(\mu \mathrm{mol} / \mathrm{l})\end{array}$ & $63.4 \pm 2.6$ & $61.8 \pm 3.5$ & $70.7 \pm 5.3$ \\
\hline $\begin{array}{l}\text { Preprandial serum } \\
\text { glucose }(\mathrm{mmol} / \mathrm{l})\end{array}$ & $4.3 \pm 0.8$ & $9.6 \pm 0.5$ & $9.7 \pm 0.4$ \\
\hline $\mathrm{HbA}_{1 \mathrm{c}}(\%)$ & & $0.062 \pm 0.0009$ & $0.072 \pm 0.0007$ \\
\hline Oral antidiabetics $(n)$ & & 11 & 13 \\
\hline Insulin $(n)$ & & 9 & 19 \\
\hline Insulin (U/day) & & $48.8 \pm 1.5$ & $41.5 \pm 2.6$ \\
\hline
\end{tabular}

Data are shown as average values. For age \pm refers to the SD of the mean; and SEM for all other values. $n=$ number of subjects in a given condition; blood pressure and heart rate data refer to the sphygmomanometric and palpatory measurements in the outpatient clinic

SBP and RR variabilities
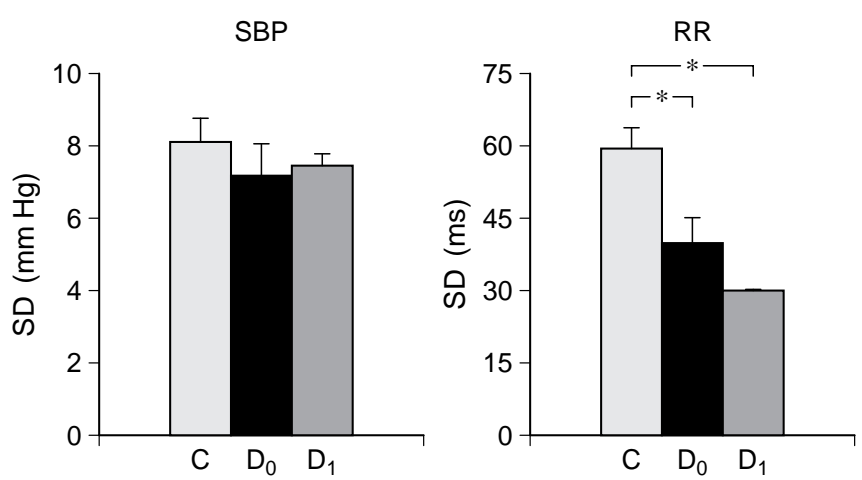

Fig. 1. Systolic blood pressure (SBP) and RR interval (RR) variability in control subjects $(\mathrm{C})$ and in diabetic patients with no or with autonomic dysfunction at classical laboratory tests $\left(\mathrm{D}_{0}\right.$ and $\left.\mathrm{D}_{1}\right)$. Variability is expressed as the standard deviation (SD) of the average values of the $15 \mathrm{~min}$ recording period in the supine position. Data are shown as means \pm SEM. $* p<0.05$

of the baroreceptor-heart rate reflex, as done when changes in systolic blood pressure and RR interval are induced in the laboratory by intravenous injections of vasoactive drugs [32, 34]. The number of the $+\mathrm{RR} /+\mathrm{SBP}$ and $-\mathrm{RR} /-\mathrm{SBP}$ sequences seen over the $15 \mathrm{~min}$ period was summed and the slopes of the + RR/ + SBP and -RR/-SBP sequences were averaged.

Finally, the systolic blood pressure and RR interval signals were subjected to power spectral analysis, as described previously [35]. To this aim, systolic blood pressure and RR interval fluctuations with a period greater than $40 \mathrm{~s}$ (frequency $\leq 0.025$
$\mathrm{Hz}$ ) were removed by high-pass filtering. The filtered time series was split into contiguous segments of 256 values. Segments containing non-stationary segments were detected and excluded by the reverse arrangement test. Power spectral densities of each stationary segment were estimated after $10 \%$ cosine tapering of the raw data using a Fast Fourier Transform. For each segment the square ratio between systolic blood pressure and RR interval powers in the frequency range of $0.07 \mathrm{~Hz}$ to $0.14 \mathrm{~Hz}$ was computed any time the coherence between the systolic blood pressure and RR interval powers was over 0.5. This ratio was termed $\alpha$ coefficient and taken as an additional index of the sensitivity of the baroreceptor-heart rate reflex $[25,32]$, which was thus studied both by a time domain and a frequency domain method. Previous studies in animals have shown that the number of the $+\mathrm{RR} /+\mathrm{SBP}$ and $-\mathrm{RR} /-\mathrm{SBP}$ sequences, the slope of both sequences and the $\alpha$ coefficients all depend on the integrity of the sino-aortic afferents, thereby specifically reflecting baroreceptor modulation of the sinus node $[27,32]$. Studies in man have further shown that the baroreflex impairment associated with a variety of conditions (e.g. aging, hypertension) is similarly identified by all three methods $[31,32]$ which thus provide independent but concordant estimates of the baroreflex function from the same basic signals.

Protocol and statistical analysis. Traditional assessment of autonomic neuropathy and computerized assessment of baroreflex sensitivity were obtained in two different days of the same week. The order of the traditional compared to computerized assessment was randomized. The investigators involved in the traditional assessment were unaware of the results of the other assessment and vice versa. Individual data were averaged to obtain mean values separately for the control group and for the D0 and D1 groups. After testing for the normal distribution of the data, between-group comparisons were made by Student's $t$-test for unpaired observations, together with application of the Bonferroni's correction for multiple comparisons. A $p<0.05$ was taken as the level of statistical significance. Unless otherwise indicated throughout the text the symbol \pm refers to the SE of the mean.

\section{Results}

As reported under methods and shown in Table 1, the three groups of subjects (controls, D0 and D1) were comparable for age, clinic systolic blood pressure, diastolic blood pressure and heart rate values. As shown in Figure 1 the systolic blood pressure standard deviation was also similar in the three groups while the RR interval standard deviation was progressively reduced from the control to the D0 and D1 group. Compared to control subjects the number and slope of $+\mathrm{RR} /+\mathrm{SBP}$ and $-\mathrm{RR} /-\mathrm{SBP}$ sequences were markedly reduced in the D0 and even more so in the D1 patients. The number of coherent segments was only slightly and non-significantly less in D0 and D1 patients, but the $\alpha$ coefficient was also strikingly reduced in both groups.

In the diabetic patients a significant correlation was found between: 1 ) the slope of the $+\mathrm{RR} /+\mathrm{SBP}$ compared to the slope of the $-\mathrm{RR} /-\mathrm{SBP}$ sequence (correlation coefficient or $r=0.61$ ); 2) the slope of the $+\mathrm{RR} /+\mathrm{SBP}$ segment compared to the $\alpha$ 
Number of sequences
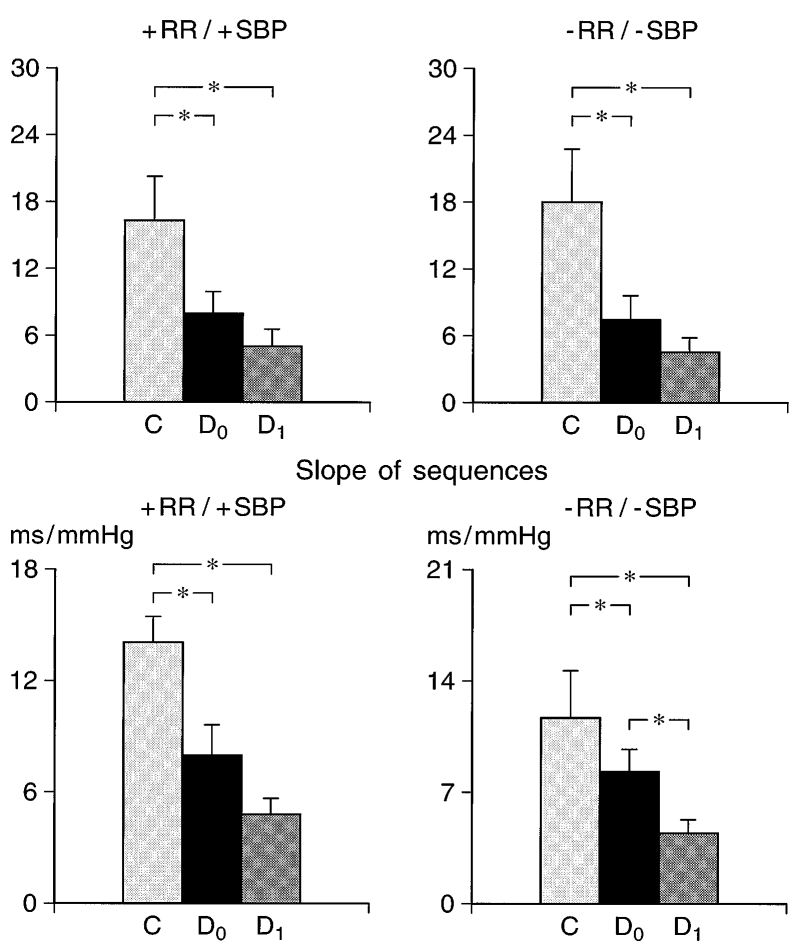

Frequency domain analysis

$\%$ of coherent segments
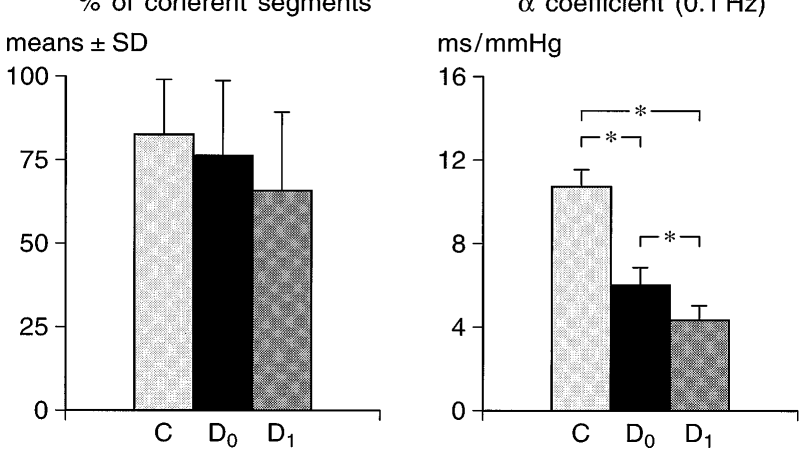

Fig. 2. Time- and frequency-domain estimates of the baroreceptor-heart rate reflex in the subjects from Figure 1. C, control subjects; $\mathrm{D}_{0}$ and $\mathrm{D}_{1}$, diabetic patients with no or autonomic dysfunction at classical laboratory tests. ${ }^{*} p<0.05$

Fig.3. Percent reduction of RR interval standard deviation, number and slope of $+\mathrm{RR} /+\mathrm{SBP}$ and $-\mathrm{RR} /-\mathrm{SBP}$ sequences, percentage of coherent segments and $\alpha$ coefficient in the $D_{0}$ and $\mathrm{D}_{1}$ as compared to $\mathrm{C}$ group coefficient $(r=0.78)$; and 3$)$ the slope of the $-\mathrm{RR} /$ -SBP sequence compared to the $\alpha$ coefficient $(r=0.61)$. A significant (although less close) correlation was also found between the standard deviation of $\mathrm{RR}$ interval and the slope of $+\mathrm{RR} /+\mathrm{SBP}$, the slope of $-\mathrm{RR} /-\mathrm{SBP}$ sequences and the $\alpha$ coefficient ( $r=0.52,0.37$ and 0.54 respectively, $p<0.01$ for all). Compared to controls the reduction in the RR interval standard deviation was less than the reduction in the number and slope of $+\mathrm{RR} /+\mathrm{SBP}$ sequences, the number and slope of $-\mathrm{RR} /-\mathrm{SBP}$ sequences and the $\alpha$ coefficient. This was the case both in the D0 and the D1 groups (Fig. 3).

\section{Discussion}

In 20 patients with diabetes mellitus, the classical "Ewing" tests failed to identify any evidence of autonomic neuropathy. In these subjects, however, time domain and frequency domain analysis of continuous systolic blood pressure and RR interval signals showed that the "spontaneous" baroreceptor modulation of the sinus node was much less effective than the modulation seen in a group of age-matched controls, although still more effective than that seen in other 32 diabetic patients with abnormal "Ewing" tests. Thus, time domain and frequency domain analysis of blood pressure and heart rate aimed at characterizing "spontaneous" baroreceptor modulation of the sinus node provides a more sensitive diagnosis of autonomic dysfunction than classical laboratory manouvers, thereby allowing abnormalities of autonomic cardiac modulation to be identified at an early stage and quantified in a graded fashion. This can be obtained by a short non invasive recording of blood pressure and ECG in the supine patients, i.e. under conditions most suitable for routine outpatient evaluation.

Several other results of our study deserve to be mentioned. One, blood pressure standard deviation was similar in control subjects, in D0 and D1 patients, which indicates that blood pressure variability is not a sensitive marker of autonomic damage in diabetes mellitus, at least when short recordings in the supine position are obtained. Two, R-R interval standard deviation showed a progressive reduction from control

$\Delta \mathrm{D}_{1}$

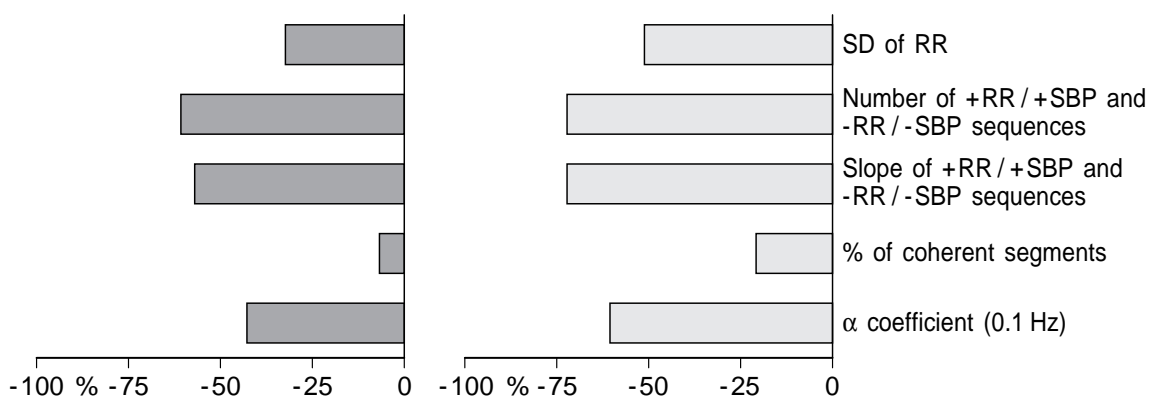


subjects to D0 and D1 patients, indicating that, in contrast, supine heart rate variability has a more important diagnostic value for this diabetic complication. Three, in D0 and D1 patients the autonomic impairment was more clear and consistent when quantified by multivariate analysis techniques than when separately focusing on blood pressure or RR interval variability alone. This is exemplified by previous data obtained through transfer function analysis between respiration and heart rate fluctuations, which has provided an accurate description of the features of autonomic cardiac control in these patients [36], and by the data obtained in our study by use of another multivariate approach, i.e. through time and frequency domain estimates of baroreflex. Thus, estimation of baroreflex cardiac modulation by joint analysis of blood pressure and RR interval fluctuations seems to be more sensitive in the early detection of autonomic cardiac neuropathy occurring in diabetes than the quantification of RR interval variability, the two measures showing only a limited correlation. The latter is also, in turn, better than traditional autonomic tests. This may be the case because, as shown by several studies, RR interval variability depends on neural but to some extent also on non-neural influences [37].

Finally, the time and frequency domain methods to quantify the baroreceptor-heart rate reflex provided superimposable data in both D0 and D1 patients. Furthermore, with the time domain method the sequence number was able to detect early autonomic dysfunction as effectively as the sequence slope. This means that the joint analysis of blood pressure and RR interval fluctuations can be limited to one method and that whenever the sequence method is selected the analysis can only consist in the calculation of how many times systolic blood pressure and RR interval display a baroreflex-type pattern. Thus the procedure necessary to obtain easily an early diagnosis of autonomic cardiac dysfunction in diabetes mellitus can indeed be so simple as to be compatible with routine procedures of an outpatient clinic.

\section{References}

1. Ewing DJ, Campbell IW, Clarke BF (1980) The natural history of diabetic autonomic neuropathy. Q J Med 49: 95-108

2. Watkins PJ, Macday JD (1980) Cardiac denervation in diabetic neuropathy. Ann Intern Med 92: 304-307

3. Rathmann W, Ziegler D, Jahnke M, Haastert B, Gries FA (1993) Mortality in diabetic patients with cardiovascular autonomic neuropathy. Diabet Med 10: 664-671

4. Navarro X, Kennedy WR, Loewenson RB, Sutherland DER (1990) Influence of pancreas transplantation on cardiorespiratory reflexes, nerve conduction and mortality in diabetes. Diabetes 39: 802-806

5. Kahn JK, Sisson JC, Vinik AI (1987) QT interval prolongation and sudden cardiac death in diabetic autonomic neuropathy. J Clin Endocrinol Metab 64: 751-754
6. Sampson MJ, Wilson S, Karagiannis P, Edmonds ME, Watkins PJ (1990) Progression of diabetic neuropathy over a decade in insulin-dependent diabetics. Q J Med 75: 635-646

7. O'Brien IA, McFadden JP, Corral RJ (1991) The influence of autonomic neuropathy on mortality in insulin-dependent diabetes. Q J Med 79: 495-502

8. Page MM, Watkins PJ (1978) Cardiorespiratory arrest and diabetic autonomic neuropathy. Lancet i: 14-16

9. Ewing DJ, Campbell IW, Clarke BF (1976) Mortality in diabetic autonomic neuropathy. Lancet i: 601-603

10. Hathaway DK, El-Gebely S, Cardoso SS, Elmer DS, Gaber AO (1995) Autonomic cardiac dysfunction in diabetic transplant recipients succumbing to sudden cardiac death. Transplantation 59: 634-637

11. Reichard P, Phil M (1994) Mortality and treatment side effects during long term intensified conventional insulin treatment in the Stockholm Diabetes Intervention Study. Diabetes 43: 313-317

12. Ewing DJ, Clarke BF (1982) Diagnosis and management of diabetic autonomic neuropathy. Br Med J 285: 916-918

13. Elisberg EI (1963) Heart rate response to the Valsalva maneuver as a test of circulatory integrity. JAMA 186 : 200-205

14. Eckberg DL (1980) Parasympathetic cardiovascular control in human disease: a critical review of methods and results. Am J Physiol 239:H581-H593

15. Sundkvist G, Lija B, Almer LO (1980) Abnormal diastolic blood pressure and heart rate reaction to tilting in diabetes mellitus. Diabetologia 19: 433-438

16. Ewing DJ, Campbell IW, Murray A, Neilson JMM, Clarke BF (1978) Immediate heart rate response to standing:simple test for autonomic neuropathy in diabetes. Brit Med J I: $145-147$

17. Consensus Statement (1995) Standardizing measures in diabetic neuropathy. Diabetes Care 18 [Suppl 1]: 59-82

18. Ewing DL, Irving GB, Kerr F, Wildsmith J, Clarke BF (1974) Cardiovascular response to sustained handgrip in normal subjects and in patients with diabetes mellitus: a test of autonomic function. Clin Sci Mol Med 46: 295-306

19. Hines EA, Brown EGE (1936) The cold pressor test for measuring the reactivity of the blood pressure: data concerning 571 normal and hypertensive patients. Am Heart J 11: $1-9$

20. Parati G, Pomidossi G, Ramirez AJ, Cesana B, Mancia G (1985) Variability of the haemodynamic responses to laboratory tests employed in assessment of neural cardiovascular regulation in man. Clin Sci 69: 533-540

21. Akselrod S, Gordon D, Ubel FA, Shannon DC, Barger AC, Cohen RJ (1981) Power spectrum analysis of heart rate fluctuation: a quantitative probe of beat to beat cardiovascular control. Science 213: 220-222

22. Freeman R, Saul JP, Roberts MS, Berger RD, Broadbridbe C, Cohen RJ (1991) Spectral analysis of heart rate in diabetic autonomic neuropathy. A comparison with standard tests of autonomic function. Arch Neurol 48: 185-190

23. Bernardi L, Rossi M, Soffiantino F, Marti G, Ricordi L, Finardi G, Fratino P (1989) Cross correlation of heart rate and respiration versus deep breathing. Assessment of new test of cardiac autonomic function in diabetes. Diabetes 38: 589-596

24. Robbe HWJ, Mulder LJM, Ruddel H, Langewitz WA, Veldman JBP, Mulder G (1987) Assessment of baroreceptor reflex sensitivity by means of spectral analysis. Hypertension 10: 538-543

25. Pagani M, Somers V, Furlan R et al. (1988) Changes in autonomic regulation induced by physical training in mild hypertension. Hypertension 12: 600-610 
26. Di Rienzo M, Bertinieri G, Mancia G, Pedotti A (1985) A new method for evaluating the baroreflex role by a joint pattern. Analysis of pulse interval and systolic blood pressure series. Med Biol Eng Comp 23 [Suppl I]: 313-314

27. Bertinieri G, Di Rienzo M, Cavallazzi A, Ferrari AU, Pedotti A, Mancia G (1988) Evaluation of baroreceptor reflex by blood pressure monitoring in unanesthetized cats. Am J Physiol 254: H377-H383

28. Parati G, Di Rienzo M, Bertinieri G et al. (1988) Evaluation of the baroreceptor-heart rate reflex by 24-hour intraarterial blood pressure monitoring in humans. Hypertension 12: 214-222

29. Parati G, Casadei R, Groppelli A, Di Rienzo M, Mancia G (1989) Comparison of finger and intra-arterial blood pressure monitoring at rest and during laboratory testing. Hypertension 13: 647-655

30. Omboni S, Parati G, Frattola A, Mutti E, Di Rienzo M, Castiglioni P, Mancia G (1993) Spectral and sequence analysis of finger blood pressure variability: Comparison with analysis of intra-arterial recordings. Hypertension 22: 22 26

31. Parati G, Frattola A, Di Rienzo M, Castiglioni P, Pedotti A, Mancia G (1995) Effects of aging on 24 hour dynamic baroreceptor control of heart rate in ambulant subjects. Am J Physiol 268: H1606-H1612
32. Parati G, Di Rienzo M, Castiglioni P, Frattola A, Omboni S, Pedotti A, Mancia G (1995) Daily-life baroreflex modulation: new perspectives from computer analysis of cardiovascular signals. In: Di Rienzo M, Mancia G, Parati G, Pedotti A, Zanchetti A (eds) Computer analysis of cardiovascular signals. IOS Press, Amsterdam pp 209-218

33. Smyth HS, Sleight P, Pickering GW (1969) Reflex regulation of arterial pressure during sleep in man: a quantitative method of assessing baroreflex sensitivity. Circ Res 24: 109-121

34. Mancia G, Mark AL (1983) Arterial baroreflexes in humans. In: Shepherd JT, Abboud FM (eds) Handbook of Physiology, sect 2, The Cardiovascular System IV, Vol 3, part 2. American Physiological Society, Bethesda pp 755793

35. Di Rienzo M, Castiglioni P, Mancia G, Parati G, Pedotti A (1989) 24 hour sequential spectral analysis of arterial blood pressure and pulse interval in free moving subjects. IEEE Trans Biomed Eng 36: 1066-1074

36. Freeman R, Cohen R, Saul P (1995) Transfer function analysis of respiratory sinus arrhythmia: a measure of autonomic function in diabetic neuropathy. Muscle Nerve 18: 74-84

37. Bernardi L, Keller F, Sanders M, Reddy PS, Griffith B, Meno F, Pinsky MR (1989) Respiratory sinus arrhythmia in the denervated human heart. J Appl Physiol 67: 1447-1455 